\title{
Exercises in Clinical Reasoning Putting Schemas to the Test: An Exercise in Clinical Reasoning
}

\author{
Daniel J. Minter, $M D^{7}$, Reza Manesh, $M D^{2}$, Patricia Cornett, $M D^{3}$, and Rabih M. Geha, MD ${ }^{1,4}$ \\ 'Department of Medicine, University of California, San Francisco, CA, USA; ${ }^{2}$ Department of Medicine, Johns Hopkins University School of Medicine, \\ Baltimore, MD, USA; ${ }^{3}$ Division of Hematology/Oncology, University of California, San Francisco, CA, USA; ${ }^{4}$ San Francisco VA Hospital, San Francisco, \\ CA, USA.
}

KEY WORDS: clinical reasoning; medical education.

J Gen Intern Med 33(11):2010-4

DOI: $10.1007 / \mathrm{s} 11606-018-4599-\mathrm{Z}$

(c) Society of General Internal Medicine 2018

\begin{abstract}
$\mathrm{A}$ 70-year-old previously healthy man presented to the emergency department with 6 weeks of fatigue, fevers, and chills. He had abdominal fullness, early satiety, and 20-pound weight loss over the preceding 8 months. He did not have headache, night sweats, cough, dyspnea, diarrhea, dysuria, or rash. His family noticed yellowing of his skin and eyes over the previous week. He had immigrated from Vietnam to the USA 30 years prior. He lived in Northern California and had traveled to Minnesota 1 month earlier. He previously worked as a landscaper and had been retired for 10 years. He denied tobacco, alcohol, or recreational drug use.
\end{abstract}

Early satiety usually represents pathology within the stomach, which may be from external compression (e.g., splenomegaly), impaired motility (e.g., gastroparesis), or more commonly, intraluminal disease (e.g., peptic ulcer disease). Yellowing of the skin usually implies hyperbilirubinemia. Elevations in indirect bilirubin result from hemolysis, impaired hepatic uptake (e.g., congestive heart failure), or deficient glucuronidation (e.g., Gilbert's syndrome). Direct hyperbilirubinemia develops from impaired hepatic excretion and is further characterized as extrahepatic or intrahepatic (e.g., viral hepatitis). Lifethreatening causes of jaundice include hemolysis, cholangitis, decompensated cirrhosis, and acute liver failure.

A rapid evaluation for ascending cholangitis is in order though the time course is more suggestive of an indolent infection or a non-infectious cause of fever and jaundice (e.g., IgG4related disease). Mycobacterium tuberculosis, Burkholderia pseudomallei (meliodosis), Talaromyces marneffei, and

In this series, a clinician extemporaneously discusses the diagnostic approach (regular text) to sequentially presented clinical information (bold). Additional commentary on the diagnostic reasoning process (italics) is integrated throughout the discussion.

Received February 23, 2018

Revised May 22, 2018

Accepted July 13, 2018

Published online July 31, 2018
Histoplasma capsulatum are prevalent infections in Vietnam that can present with a subacute febrile hepatitis. Diffuse hepatic infiltration by these granulomatous infections leads to intrahepatic cholestasis; jaundice may also result from lymphadenopathy compressing the external biliary tree. Liver abscess caused by hypermucoviscous Klebsiella pneumoniae is more common in Asian patients, but cholestasis would be unlikely unless the abscess was compressing the large caliber bile ducts.

In approaching diagnostic problems, clinicians first construct a problem representation (PR) - a one-sentence summary that highlights the key features of the case-and then search their mental library of illness scripts (IS) for an explanatory diagnosis. ${ }^{1}$ These IS are collections of data that summarize a clinician's knowledge about a given disease or disorder. ${ }^{2}$ Occasionally, a PR immediately activates an IS. However, more complex clinical scenarios may lead to PRs that do not reflexively trigger a single diagnosis. In such instances, the use of diagnostic schemas - conceptual frameworks that provide a structured approach to an abnormality - can facilitate a more analytic method of problem solving. ${ }^{3}$ As an example, the discussant uses his schema for causes of jaundice, which in addition to providing a structure to think through a single problem, has the added benefit of containing a "do not miss" diagnostic checklist. In creating a structured and more manageable approach to problem solving, schemas reduce the cognitive load and provide increased bandwidth for more effective reasoning. ${ }^{4}$

In this case, the discussant is faced with a challenging diagnostic scenario: an elderly man from Vietnam with prolonged fevers, jaundice, and early satiety. While this $P R$ raises a range of diagnostic possibilities, it does not immediately clinch an obvious explanatory diagnosis. In response, the discussant meticulously applies diagnostic schemas to the patient's various complaints - early satiety, jaundice, and prolonged fevers. By employing schemas for each individual complaint in parallel, he is able to consider all of the available data without prematurely discarding potentially important information.

His temperature was $38.2^{\circ} \mathrm{C}$, heart rate $60 \mathrm{bpm}$, blood pressure $118 / 81 \mathrm{mmHg}$, respiratory rate $18 \mathrm{times} / \mathrm{min}$, and oxygen saturation $96 \%$ while breathing ambient air. He had scleral icterus and jaundiced skin. There was no lymphadenopathy. His cardiopulmonary exam was 
normal. He had mild right upper quadrant tenderness. There was no hepatosplenomegaly, spider nevi, palmar erythema, or distended abdominal veins.

The white cell count was $2700 / \mathrm{mm}^{3}$, hemoglobin $12.0 \mathrm{~g} /$ $\mathrm{dL}$, and platelet count $61,000 / \mathrm{mm}^{3}$. His metabolic panel was normal. His aspartate aminotransferase (AST) was $72 \mathrm{U} / \mathrm{L}$ (normal, 17-42), alanine aminotransferase (ALT) $172 \mathrm{U} / \mathrm{L}$ (normal, 12-60), alkaline phosphatase $449 \mathrm{U} / \mathrm{L}$ (normal, 31-95), and total bilirubin $10.1 \mathrm{mg} / \mathrm{dL}$ (normal, 0.2-1.3) with a direct bilirubin of $8.0 \mathrm{mg} / \mathrm{dL}$. The prothrombin time was $19.5 \mathrm{~s}$ (normal, 11.8-15.8), activated partial thromboplastin time $48 \mathrm{~s}$ (normal, 22.6-34.5), and INR 1.9. The albumin was $2.5 \mathrm{~g} / \mathrm{dL}$. A CT of the abdomen with contrast demonstrated wall thickening of the stomach and the ascending colon with associated fat stranding, mild splenomegaly, and hepatic steatosis. There was no biliary dilation.

Coagulopathy in the presence of hepatitis is consistent with acute liver injury. This merits vigilance for changes in mental status that may herald acute liver failure. Low vitamin K may also be contributing to the elevated PT and PTT. This can result from reduced intake (malnutrition), impaired absorption (cholestasis), decreased vitamin $\mathrm{K}$-synthesizing gut flora (antibiotics), or direct effect of medications (warfarin). A rapid improvement in the INR with vitamin $\mathrm{K}$ therapy supports these considerations over hepatic synthetic dysfunction or consumptive coagulopathy.

The absence of biliary dilation on CT makes an intrahepatic cause of cholestasis more likely, although more sensitive imaging (e.g., MRCP) may detect subtle extrahepatic disease such as a biliary stricture. Causes of hepatocellular injury (e.g., viral hepatitis) are possible as the bilirubin level normalizes in a delayed manner following a primary hepatocellular insult. Primary biliary cholangitis causes intrahepatic cholestasis, but not fever. Medications (e.g., amoxicillin) and infiltrative hepatopathies such as sarcoidosis and lymphoma may cause fever and intrahepatic cholestastis.

Pancytopenia may result from splenic sequestration. Evidence to support the most common cause of splenomegaly, portal hypertension, is lacking (no ascites, lower extremity edema, or caput medusae). Thus, the cause of splenomegaly is likely from pathology within the reticuloendothelial system (bone marrow and spleen). Bone marrow diseases might result from reduced raw materials (e.g., B12 deficiency), stem cell diseases (e.g., aplastic anemia, myelodysplasia), or myelophthisis, which refers to infiltration by malignant cells (e.g., lymphoma), granulomas (e.g., tuberculosis), aberrant macrophages (e.g., Langerhans histiocytosis), or fibrosis (e.g., myelofibrosis). Concomitant splenomegaly and bone marrow disease (Fig. 1) usually reflects the presence of a myelophthisic process. Pancytopenia may also be destructive in nature and result from autoantibodies (e.g., lupus) or infections (e.g., human granulocytic anaplasmosis).

The discussant continues to individually apply his diagnostic schemas to the newly presented data-outlining his approach to coagulopathy, splenomegaly, and bone marrow diseases. Additionally, he creates a schema for combined abnormalities (e.g., splenomegaly and bone marrow disease) to narrow the field of diagnostic possibilities. In doing so, he is able to quickly consider conditions that could provide a parsimonious explanation of the patient's clinical picture (i.e., a myelophthisic process). He reflects back to his broader schema for pancytopenia (sequestration, bone marrow pathology, and peripheral destruction), however, in order to consider all potential etiologies and avoid premature closure.

The patient was administered piperacillin/tazobactam. Endoscopic retrograde cholangiopancreatography (ERCP) demonstrated several small filling defects in the midportion of the common bile duct thought to represent small stones. No mass or stricture was seen and a sphincterotomy was performed. Blood and urine cultures remained sterile. His fever persisted and antimicrobial coverage was broadened to vancomycin, meropenem, doxycycline, and fluconazole. A subsequent tagged WBC scan was negative for localizing infection or inflammation. Over the following 5 days, his AST increased to $158 \mathrm{U} / \mathrm{L}$ and ALT to $221 \mathrm{U} / \mathrm{L}$. His alkaline phosphatase remained elevated at $368 \mathrm{U} / \mathrm{L}$. Hepatitis A IgG was positive with a negative IgM. Hepatitis B surface antibody, surface antigen, and viral load by PCR were negative, while the core antibody was positive. Hepatitis $\mathrm{C}$ antibody was negative. A CT-guided transhepatic liver biopsy showed cholestasis without evidence of cirrhosis or inflammation.

He developed oliguric kidney failure that required renal replacement therapy. Urinalysis showed 9 white blood cells and 3 red blood cells per high-power field (normal, $0-2$ ), and urine microscopy revealed muddy brown casts. He developed progressive cytopenias and coagulopathy (hemoglobin 7.1, platelets 34,000, and INR 2.7), which required periodic packed red blood cell, platelet, and fresh frozen plasma transfusions. On hospital day 10, the serum Epstein Barr virus (EBV) PCR returned positive at greater than 1 million copies. He was given a presumptive diagnosis of $\mathrm{EBV}$-associated acute liver failure and transferred to a tertiary care center.

An aggressive search for cholangitis in a patient with fever and jaundice is prudent but the ERCP findings and subsequent clinical course exclude this diagnosis. Mild hematuria and pyuria may suggest glomerulonephritis and interstitial nephritis, respectively, though renal failure in a critically ill patient is often due to acute tubular necrosis (ATN) and is supported by the presence of muddy brown casts. Importantly, ATN is often a non-specific complication of critical illness rather than a signature of an underlying disease process. A prolonged febrile syndrome and hepatitis may occur in acute infectious mononucleosis, but multi-organ failure is unusual. EBV reactivation might develop in any critical illness. The marked viremia in this case is more consistent with underlying EBVassociated malignancy. 


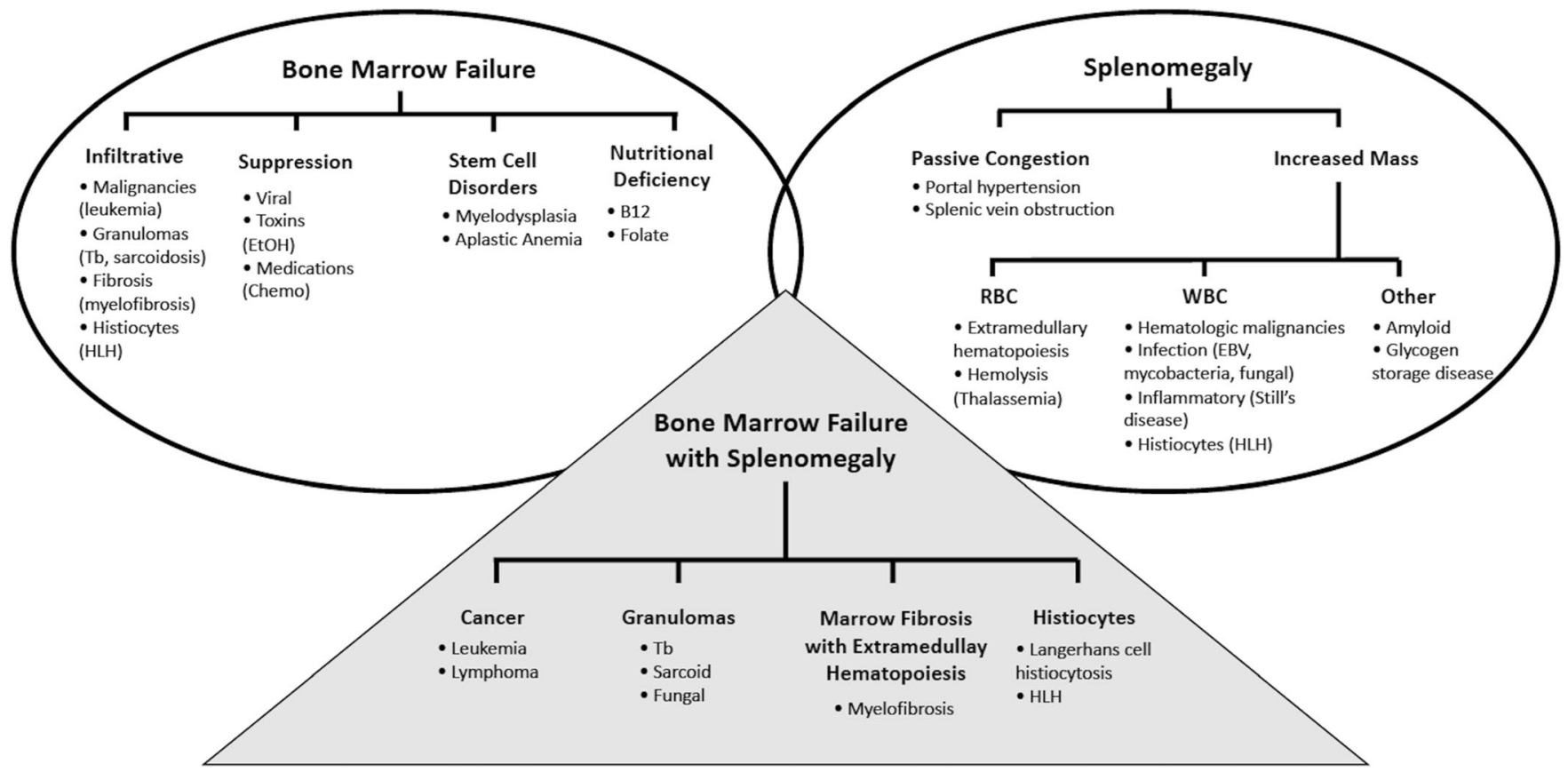

Figure 1 The clinician provides his individual schemas for bone marrow failure and splenomegaly. In an effort to identify a parsimonious diagnosis, he references a combined schema for the two findings. Tb, tuberculosis; EtOH, alcohol; B12, cobalamin; chemo, chemotherapy; EBV, Epstein-Barr virus; HLH, hemophagocytic lymphohistiocytosis.

The worsening inflammatory syndrome despite broadspectrum antibiotic therapy makes a pyogenic bacterial infection unlikely. The patient has features of hemophagocytic lymphohistiocytosis (HLH), an inflammatory syndrome characterized by hepatopathy, splenomegaly, cytopenias, and phagocytosis of red blood cells by macrophages. In adults, HLH often develops due to an underlying infection, autoimmune disease, or malignancy. Neoplasms leading to HLH are usually hematologic, although solid tumors such as nasopharyngeal carcinoma, with a higher incidence in East Asia, can also trigger $\mathrm{HLH}$.

Acute oliguric renal failure is a notable development in the case but the discussant does not deploy a schema for this condition. Instead, after a brief consideration of a short list of possibilities, he determines that this is likely a bystander and focuses on more diagnostically rich information. The discussant's understanding of the clinical findings has matured through his employment of multiple diagnostic schemas (i.e., non-localizing fever despite antibiotics, cytopenias with splenomegaly, and intrahepatic cholestasis). These features are included in his evolving $P R$, which he now recognizes as meeting many of the clinical criteria for HLH. At this point, he utilizes a schema for precipitants of HLH, directing his search towards underlying triggers.

On arrival, his temperature was $39.1^{\circ} \mathrm{C}$ and remainder of exam was unchanged. An automated smear revealed $63.3 \%$ neutrophils, $14.8 \%$ lymphocytes, $2.1 \%$ immature granulocytes, and nucleated red blood cells. His serum ferritin was $3823 \mu \mathrm{g} / \mathrm{L}$ (normal, 30-530), iron $94 \mu \mathrm{g} / \mathrm{dL}$ (normal, 42-175), transferrin $92 \mathrm{mg} / \mathrm{dL}$ (normal, 182-
360), and transferrin saturation 73\% (normal, 10-47). Serum haptoglobin was $<6 \mathrm{mg} / \mathrm{dL}$, reticulocyte count $58,600 / \mathrm{mm}^{3}$ (normal, 29,000-121,400), lactate dehydrogenase $329 \mathrm{U} / \mathrm{L}$ (normal, 102-199), and fibrinogen $173 \mathrm{mg} /$ dL (normal, 202-430). The C-reactive protein was $70.2 \mathrm{mg} / \mathrm{L}$ (normal, $<6.3$ ) and serum triglycerides $324 \mathrm{mg} / \mathrm{dL}$ (normal, <200). Tests for antinuclear and anti-smooth muscle antibodies were negative. CMV IgG was positive with a negative PCR. He had a negative EBV IgM but positive IgG. Serology and urinary antigen testing for histoplasmosis were negative.

A leukoerythroblastic reaction- defined as the presence of nucleated red blood cells, tear drop cells, and white blood cell precursors - invariably signals an infiltrative bone marrow syndrome. A low haptoglobin raises the possibility of intravascular hemolysis, although impaired hepatic function and ineffective erythropoiesis may be explanatory.

The elevated transferrin saturation is consistent with iron overload. Hemochromatosis is uncommon in Vietnam. Acquired causes of iron overload are more likely; these include a variety of hepatic (e.g., chronic hepatitis C) and hematologic diseases (e.g., myelodysplasia). Ferritin is an acute phase reactant and therefore is non-specific; however, the degree of hyperferritinemia supports the presence of HLH. The positive EBV IgG excludes acute EBV and reinforces the need to search for an underlying EBV-associated malignancy to explain his EBV viremia.

The discussant continues to apply diagnostic schemas to the individual components of the new data. He identifies the 
abnormal hematology studies as a leukoerythroblastic reaction implying a bone marrow syndrome. He discusses the iron studies and briefly details his schema for iron overload, placing less weight on possibilities inconsistent with his understanding of the case (e.g., hemochromatosis). As his many parallel schemas appear to converge on a diagnosis of HLH, his PR matures and becomes less malleable. He carefully evaluates the newly presented data to see how it fits within his understanding of the current illness (e.g., identifying the elevated ferritin and pancytopenia as being consistent with the presence of HLH) rather than letting it redirect his thinking entirely (e.g., exploring a schema for hemolysis because of the low haptoglobin).

A soluble IL-2 receptor level was $12,520 \mathrm{pg} / \mathrm{mL}$ (normal, $\leq 1033$ ). A bone marrow biopsy resulted in a "dry tap" (unable to obtain a bone marrow aspirate).

The elevated soluble IL-2 receptor in combination with the persistent fever, hyperferritinemia, hypertriglyceridemia, cytopenias, and splenomegaly fulfills the diagnostic criteria for HLH. A dry tap signals significant bone marrow disease either due to fibrosis (e.g., myelofibrosis) or extensive hypercellularity (e.g., AML).

Steroid therapy was initially held given the possibility of HLH being triggered from an undiagnosed infection. However, upon further clinical deterioration, empiric steroids were initiated and, within hours, his fevers abated. The bone marrow biopsy revealed rare hemophagocytic histiocytes on a background of hypercellular bone marrow (Fig. 2a). Large areas of necrosis with an interstitial EBVpositive lymphoid infiltrate composed of predominantly malignant-appearing NK cells demonstrating an aberrant immunophenotype were noted (Fig. 2b).
The erythrophagocytosis further supports the presence of $\mathrm{HLH}$, as does the improvement with steroid therapy. The bone marrow findings are diagnostic of NK-T cell leukemia, a rare, aggressive, EBV-positive neoplasm seen predominantly in men of Asian or South American descent and associated with a high frequency of HLH.

The liver biopsy from the outside hospital was reexamined and demonstrated a sinusoidal EBV-positive NK cell infiltrate with areas of necrosis and hemophagocytosis, further supporting the diagnosis of an aggressive NK cell leukemia with associated HLH. He was briefly treated with etoposide and dexamethasone, but given his poor prognosis, he was ultimately transitioned to comfort care and discharged home with hospice care.

\section{COMMENTARY}

Diagnostic schemas are cognitive frameworks that provide a structured approach to different clinical scenarios - chief complaints (e.g., altered mental status), abnormal laboratory values (e.g., anemia), or physical findings (e.g., neuromuscular weakness). ${ }^{3}$ In medical education, schemas help learners understand key concepts, and further serve as invaluable tools in clinical reasoning. By providing a conceptual framework coded in long-term memory, schemas also facilitate an analytical approach to complex scenarios.

As in this case, a patient may present with many seemingly unrelated findings that fail to activate a specific illness script. In such circumstances, the use of multiple parallel schemas helps the reasoner methodically consider all the data before prematurely dismissing an abnormality. By serving as a checklist for "cannot miss" conditions, schemas may also help clinicians avoid life-threatening diagnostic errors; for example, a schema for fatal causes of abdominal pain might include

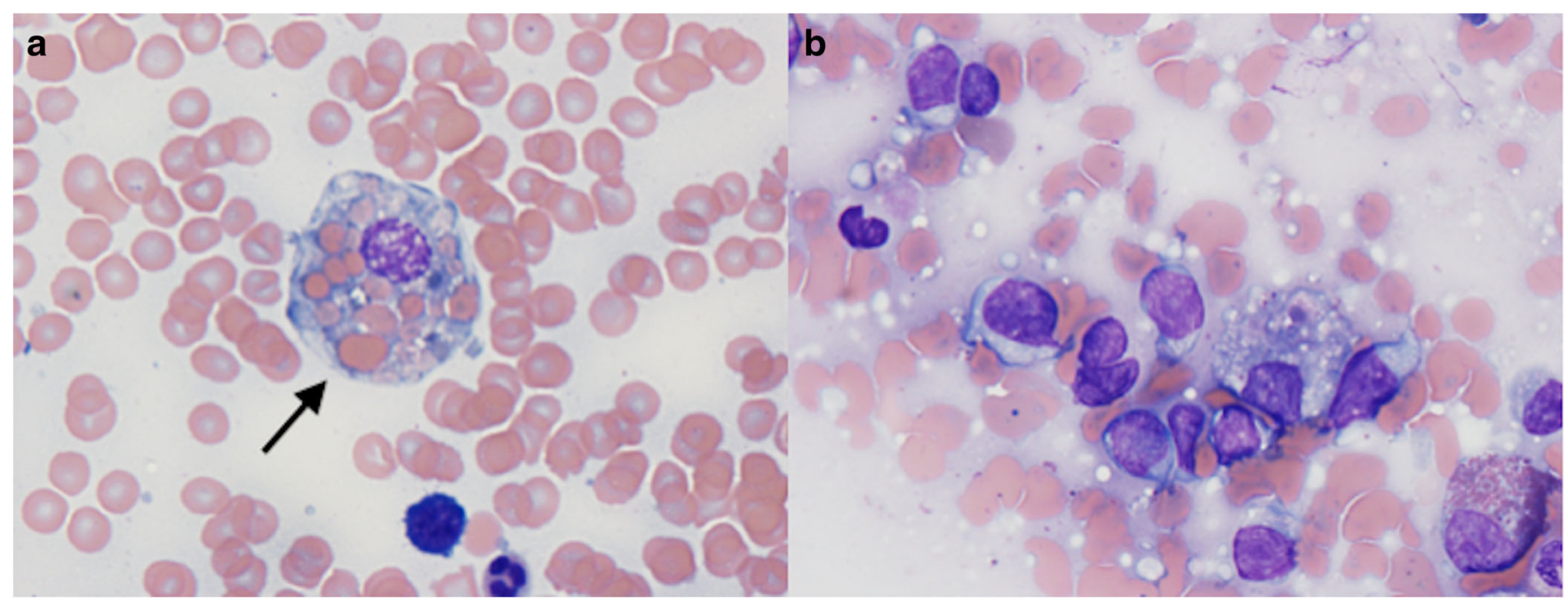

Figure 2 Bone marrow aspirate at $\times 100$. a A macrophage undergoing erythrophagocytosis with multiple intracellular erythrocytic elements (arrow). b A touch preparation of the biopsy demonstrating multiple neoplastic NK cells. 
obstruction, perforation, and ischemia. Additionally, multiple schemas can be combined to rapidly search for a unifying diagnosis to explain a patient's presentation (Fig. 1). Finally, by relying on familiar schemas, clinicians can consider a wider range of possible etiologies for the primary abnormality while maintaining cognitive space to detect subtle aberrations in the rest of the data.

A physician's schemas are often uniquely developed and evolve with time. Often originating in clinical training, schemas mature and become more nuanced with experience. Inevitably, this leads to heterogeneity of diagnostic approaches across the profession. Whereas one person's schema for splenomegaly may be based on the pathophysiologic mechanism (i.e., passive congestion versus increased cellular or molecular mass), another's might highlight the most common etiologies (i.e., congestion, infection, malignancy, autoimmune diseases, and hemolysis). In each case, the clinician grows comfortable with their version of tackling a clinical problem.

Yet with such a variety of different schemas in use, some must inherently allow the reasoner to reach the diagnosis in fewer steps and with more accuracy. Should this heterogeneity be the subject of rigorous study? Much could be learned from other logic-based disciplines, like computer science, where the performance of different algorithms is evaluated to reduce computational complexity. ${ }^{5}$ If such testing were applied to diagnostic schemas, approaches that reach the ultimate diagnosis more often and with the fewest number of steps could be identified. This may prove important as the medical field becomes increasingly focused on eradicating overtesting and containing healthcare expenditures. At the very least, it highlights the potential to apply the methods of evidence-based medicine, which has revolutionized our understanding of therapeutic efficacy, to the essential and universal clinical skill of diagnostic reasoning.

\section{CLINICAL TEACHING POINTS}

\section{Epstein-Barr Virus (EBV) viremia}

- Infection with EBV is nearly ubiquitous, with more than $90 \%$ of people estimated to be infected worldwide. $^{6}$ EBV has a strong tropism for B lymphocytes but can also infect NK cells and T lymphocytes. This rare event is strongly associated with development of EBV-associated HLH, NK/T cell lymphomas/leukemias, and chronic active EBV infection. ${ }^{7,8}$

2. Hemophagocytic lymphohistiocytosis (HLH)

- HLH is a rare, life-threatening syndrome characterized by pathologic immune activation leading to multi-organ damage. ${ }^{7}$ It can occur as a primary (e.g., genetic) or secondary disorder, with the latter most commonly triggered by infections, autoimmune conditions, and malignancies.

3. Natural killer (NK)/T cell lymphomas and leukemia

- NK/T cell lymphomas are rare, aggressive EBVassociated neoplasms that usually present with lesions in the nose or nasopharynx. Extra-nasal NK/ $\mathrm{T}$ cell lymphoma (e.g., originating in the GI tract, skin, or testes) is less common and carries a worse prognosis. ${ }^{9}$ Rarely, these malignancies can present in a fulminant fashion with involvement of the bone marrow and peripheral blood. These cases are classified as NK cell leukemias and are nearly universally fatal. ${ }^{10}$

Corresponding Author: Daniel J. Minter, MD; Department of Medicine University of California, San Francisco, CA, USA (e-mail: Daniel.Minter@ucsf.edu).

\section{Compliance with Ethical Standards:}

Conflict of Interest: Dr. Minter, Dr. Cornett, and Dr. Geha do not have any disclosures.

Dr. Manesh is supported by the Jeremiah A. Barondess Fellowship in the Clinical Transaction of the New York Academy of Medicine, in collaboration with the Accreditation Council for Graduate Medical Education (ACGME).

\section{REFERENCES}

1. Keenan CR, Dhaliwal G, Henderson MC, Bowen JL. A 43-year-old woman with abdominal pain and fever. J Gen Intern Med, 2010. 25(8): p. 874-7.

2. Jones B, Brzezinski WA, Estrada CA, Rodriguez M, Kraemer RR. A 22year-old woman with abdominal pain. J Gen Intern Med, 2014. 29(7): p. 1074-8.

3. Martin M, Manesh RS, Henderson MC, Critchfield JM. Diagnostic Scheming. J Gen Intern Med, 2015. 30(12): p. 1874-8.

4. Blissett S, Goldszmidt M, Sibbald M. Do research findings on schemabased instruction translate to the classroom? Perspect Med Educ, 2015. 4(6): p. 334-338.

5. Sedgewick R, Flajolet P. An introduction to the analysis of algorithms. 2017 [cited 2018 June 26]; Available from: http://aofa.cs.princeton.edu/ home/.

6. Freeman HR, Ramanan AV. Review of haemophagocytic lymphohistiocytosis. Arch Dis Child, 2011. 96(7): p. 688-93.

7. Ramos-Casals M, Brito-Zeron P, Lopez-Guillermo A, Khamashta MA, Bosch X. Adult haemophagocytic syndrome. Lancet, 2014. 383(9927): p. 1503-1516.

8. Fox CP, Shannon-Low C, Gothard $\mathbf{P}$, et al.. Epstein-Barr virusassociated hemophagocytic lymphohistiocytosis in adults characterized by high viral genome load within circulating natural killer cells. Clin Infect Dis, 2010. 51(1): p. 66-9.

9. Cheung MM, Chan JK, Wong KF. Natural killer cell neoplasms: a distinctive group of highly aggressive lymphomas/leukemias. Semin Hematol, 2003. 40(3): p. 221-32.

10. Tse E, Kwong YL. How I treat NK/T-cell lymphomas. Blood, 2013. 121(25): p. 4997-5005. 